\title{
Secuelas del neurodesarrollo de recién nacidos prematuros de extremadamente bajo peso y de muy bajo peso a los dos años de edad, egresados de la Unidad de Cuidados Intensivos Neonatales del Hospital Nacional Edgardo Rebagliati Martins 2009-2014
}

\author{
Carmen Fernández Sierra ${ }^{1}$, Juan Matzumura Kasano ${ }^{2}$, Hugo Gutiérrez Crespo ${ }^{3}$, Luisa Zamudio Eslava ${ }^{4}$, Giannina Melgarejo \\ García $^{5}$
}

\section{RESUMEN}

Objetivo: Describir las secuelas del neurodesarrollo de los recién nacidos prematuros de extremadamente bajo peso y de muy bajo peso a los dos años de edad, egresados de la Unidad de Cuidados Intensivos Neonatales del Hospital Nacional Edgardo Rebagliati Martins.

Materiales y métodos: Estudio descriptivo, retrospectivo, transversal en una población de 190 prematuros de extremadamente bajo peso y de muy bajo peso que nacieron durante enero 2009 a junio del 2014, egresados de la Unidad de Cuidados Intensivos Neonatales, que asistieron al programa de seguimiento. Se evaluaron el desarrollo psicomotor, hipoacusia neurosensorial, retinopatía de la prematuridad, presencia de parálisis cerebral y síndrome convulsivo.

Resultados: El promedio de peso al nacimiento fue 1.180,53 $\pm 212,40$ gramos con edad gestacional de $29,86 \pm 2,33$ semanas, y el 51,58\% fue de sexo masculino. El 42,63\% de los recién nacidos prematuros de muy bajo peso presentó retraso del desarrollo psicomotor; el 25,26\%, retinopatía; el 13,68\%, hipoacusia neurosensorial; el 3,68\%, parálisis cerebral; y el $3,68 \%$, síndrome convulsivo. El 52,27\% de los recién nacidos prematuros de extremadamente bajo peso presentó retraso en el desarrollo psicomotor; el 50\%, retinopatía; el 15,91\%, hipoacusia neurosensorial; y el 2,27\%, síndrome convulsivo. Conclusiones: El retraso del desarrollo psicomotor y la retinopatía fueron las complicaciones más importantes que presentaron los recién nacidos prematuros de extremadamente bajo peso y los recién nacidos prematuros de muy bajo a los dos años de edad.

Palabras clave: Neurodesarrollo; prematuro; recién nacido.

\section{Neurodevelopmental sequelae in premature newborns with extremely low weight and with very low weight at two years of age who left the Neonatal Intensive Care Unit of the Hospital Nacional Edgardo Rebagliati Martins 2009-2014}

\begin{abstract}
Objective: The purpose of this study is to describe the neurodevelopmental sequelae in premature newborns with extremely low weight and with very low weight at two years of age who left the Neonatal Intensive Care Unit of the Hospital Nacional Edgardo Rebagliati Martins.

Materials and methods: A descriptive, retrospective, cross-sectional study in a population of 190 premature newborns with extremely low weight and with very low weight born from January 2009 to June 2014 who left the Neonatal Intensive Care Unit and took part in the follow-up program. The psychomotor development, sensorineural hearing loss, retinopathy of prematurity, presence of cerebral palsy and convulsive syndrome were assessed.

Results: The average weight at birth was 1,180.53 \pm 212.40 grams with a gestational age of $29.86 \pm 2.33$ weeks, and $51.58 \%$ of the newborns were male. Forty-two point six three percent $(42.63 \%)$ of the premature newborns with very low weight showed retardation of psychomotor development; $25.26 \%$, retinopathy; $13.68 \%$, sensorineural hearing loss; $3.68 \%$, cerebral palsy; and 3.68\%, convulsive syndrome. Fifty-two point two seven percent $(52.27 \%)$ of the premature newborns with extremely low weight showed retardation of psychomotor development; 50\%, retinopathy; $15.91 \%$, sensorineural hearing loss; and $2.27 \%$, convulsive syndrome.

Conclusions: Retardation of psychomotor development and retinopathy were the most important complications shown by premature newborns with extremely low weight and with very low weight at two years of age.
\end{abstract}

Keywords: Neurodevelopment; premature; newborn.

1. Médico Neonatólogo, Unidad de Cuidados Intensivos Neonatales del Hospital Nacional Edgardo Rebagliati Martins.

2. Médico Gineco-Obstetra, Profesor Asociado de la Universidad de San Martín de Porres.

3. Obstetra, Magíster en Docencia e Investigación en Salud, Coordinador Académico de la Sociedad Peruana de Obstetricia y Ginecología.

4. Enfermera de la Unidad de Cuidados Intensivos Neonatales del Hospital Nacional Docente Madre Niño San Bartolomé.

5. Médico-Cirujano, Asociación para el Desarrollo de la Investigación Estudiantil en Ciencias de la Salud. 


\section{INTRODUCCIÓN}

Recientes publicaciones estiman que, actualmente a nivel mundial, nacen 15 millones de neonatos pretérminos, es decir, antes de las 37 semanas de gestación. La prematuridad es la primera causa de mortalidad neonatal en el mundo y ocupa un segundo lugar dentro de las muertes infantiles, siendo responsable de 1,1 millón de muertes anuales ${ }^{(1,2)}$. Lo anterior origina el $80 \%$ de las muertes dentro del primer mes de vida y el $98 \%$ de estas muertes neonatales ocurren en los países en desarrollo como el nuestro. La prematuridad se establece como un problema creciente de salud pública debido al incremento de técnicas de reproducción asistida y otros factores tales como la edad materna ${ }^{(3)}$.

Actualmente se define como prematuros a los infantes nacidos antes de las 37 semanas de gestación, contando desde el primer día posterior al último periodo de menstruación de la madre. Aproximadamente, el 5\% de todos los nacimientos pretérminos corresponden a los menores de 28 semanas o prematuros extremos; mientras que el $15 \%$ de 28 y menores de 32 semanas o muy prematuros; el $20 \%$ son de 32 y menores de 34 semanas o moderadamente prematuros; y el $60 \%$ restante son de 34 a 36 semanas o prematuros tardíos ${ }^{(4)}$. Debido a que esta fecha puede ser incierta o desconocida, los recién nacidos pueden ser, además, clasificados según el peso de nacimiento. El recién nacido con bajo peso al nacer es aquel cuyo peso dentro de la primera hora de nacido es menor de 2.500 gramos; cuando el peso es menor de 1.500 gramos, se considera como prematuro de muy bajo peso al nacer; $y$, extremadamente prematuro, si su peso al nacer es menor a 1.000 gramos $^{(5,6)}$.

Diferentes publicaciones reportan que ha mejorado la sobrevida en los prematuros, pero se han incrementado las secuelas o discapacidades en este grupo de recién nacidos, siendo indispensable constituir, implementar y mantener las unidades de seguimiento del recién nacido de alto riesgo, con la finalidad de continuar el manejo que se inició idealmente en la Unidad de Cuidados Intensivos Neonatales y que proseguirá luego del alta del paciente ${ }^{(7)}$.

La prematuridad no solo es un componente primordial en la muerte, sino también en otros eventos adversos, como las alteraciones del neurodesarrollo. Cualquier lesión que ocurra en el cerebro del recién nacido prematuro comprometerá un tiempo crítico de su desarrollo, ya que el cerebro inmaduro cursa por un periodo de activa mielinización ${ }^{(8)}$. Otros factores que contribuyen a las alteraciones en este grupo de recién nacidos incluyen la edad gestacional, las complicaciones respiratorias, la displasia broncopulmonar, las infecciones maternas o sepsis neonatal, entre otros. Si bien se ha logrado mejorar la sobrevida de los prematuros de muy bajo peso al nacer, la tarea pendiente es que dicha sobrevida suceda con menos morbilidades ${ }^{(9,10)}$.

La frecuencia de las alteraciones del neurodesarrollo en los prematuros de muy bajo peso al nacer es alarmante, siendo esta responsable hasta de un 50\% de las anormalidades neurológicas de la infancia, que incluyen desde las alteraciones leves de las funciones cognitivas hasta la parálisis cerebral infantil, retraso en el desarrollo psicomotor, sordera o hipoacusia neurosensorial, retinopatía de la prematuridad, que en su grado severo ocasionaría la ceguera ${ }^{(11,12)}$.

Se plantea, como uno de los desafíos de la pediatría en nuestro país, lograr que la supervivencia de estos recién nacidos sea de calidad. Las acciones dirigidas a mejorar la sobrevida sin secuelas neurológicas en el prematuro menor de 1.500 gramos tendrían un impacto favorable en la salud pública $^{(13)}$.

Aún no disponemos de investigaciones realizadas en nuestro país relacionadas al seguimiento de los prematuros, determinando la frecuencia de las alteraciones en el neurodesarrollo de neonatos de muy bajo peso al nacer.

El objetivo del estudio fue describir las secuelas del neurodesarrollo en recién nacidos de muy bajo peso a los dos años de edad, egresados de la Unidad de Cuidados Intensivos del Hospital Nacional Edgardo Rebagliati Martins entre los años 2009 y 2014.

\section{MATERIALES Y MÉTODOS}

El estudio realizado fue de tipo descriptivo, retrospectivo y de corte transversal. La población estuvo constituida por 745 recién nacidos de muy bajo peso, que ingresaron a la Unidad de Cuidados Intensivos Neonatales del Servicio de Neonatología del Hospital Nacional Edgardo Rebagliati Martins entre enero del 2009 y junio del 2014. Los criterios de inclusión establecidos para el presente estudio lo conformaron las historias clínicas de los recién nacidos prematuros con extremo bajo peso y muy bajo peso (nacidos entre enero del 2009 a junio del 2014) que completaron sus evaluaciones en las áreas de medicina física y rehabilitación, oftalmología, neurología, neurofisiología y que cumplieron los dos años de edad. Los criterios de exclusión: recién nacidos prematuros fallecidos, con presencia de malformaciones congénitas mayores, datos incompletos de sus respectivas historias clínicas, aquellos que abandonaron el seguimiento y aquellos referidos de otros hospitales.

Se confeccionó una ficha de recolección de datos tomando como referencia las publicaciones realizadas por Kawaza, Arreola-Ramírez y Barra ${ }^{(14-16)}$. La ficha estuvo conformada por cuatro dimensiones: antecedentes maternos o prenatales ( 5 ítems), características natales y postnatales (14 ítems), alteraciones neurosensoriales (2 ítems), alteración desarrollo psicomotor y neurológico (3 ítems).

En la dimensión antecedentes maternos o prenatales, se consideró la preeclampsia, el embarazo múltiple, la ruptura prematura de membranas, la corioamnionitis y el uso de medicamentos, es decir, la aplicación de corticoides prenatales. 
Secuelas del neurodesarrollo de recién nacidos prematuros de extremadamente bajo peso y de muy bajo peso a los dos años de edad, egresados de la Unidad de Cuidados Intensivos Neonatales del Hospital Nacional Edgardo Rebagliati Martins 2009-2014

La dimensión características natales y postnatales estuvo conformada por el peso al nacer, que fue agrupado en dos categorías: menor de 1.000 gramos y mayor de 1000 gramos; la edad gestacional al nacer se clasificó en semanas según fecha de última menstruación y se formaron dos grupos: menor de 28 semanas y mayor de 28 semanas; Apgar al primer minuto y Apgar a los 5 minutos; el tipo de parto: vaginal o cesárea; el género; el peso adecuado para la edad gestacional; si requirió reanimación al nacer y características postnatales; la aplicación de surfactante; si presentó sepsis precoz o tardía, displasia broncopulmonar (DBP), hemorragia intraventricular (HIV), enterocolitis necrotizante (NEC), persistencia del conducto arterioso (PCA); y días de estancia hospitalaria (mayor de 46 días).

La dimensión alteraciones neurosensoriales consideró la detección de retinopatía de la prematuridad (ROP) y la detección de la hipoacusia neurosensorial, a través de los potenciales evocados auditivos del tallo cerebral (PEATC).

La dimensión desarrollo psicomotor y alteraciones neurológicas (el síndrome epiléptico y la parálisis cerebral) fueron evaluadas según las guías de atención del Servicio de Medicina Física y Rehabilitación Pediátrica y del Servicio de Neurología Pediátrica del Hospital Nacional Edgardo Rebagliati Martins.

La validez del contenido del instrumento se realizó a través del juicio de expertos; el índice de Kappa correspondió a 0,92 . La confiablidad total del instrumento correspondió a 0,84 mediante el coeficiente de Alfa de Cronbach; asimismo, se utilizó un Análisis de Correspondencias Múltiples (ACM) para cada dimensión.
El procesamiento de la información se realizó mediante el uso del programa MS Excel. Luego del control de calidad de los datos y las variables, fueron exportados y analizados en Stata 12 trial. Se comparó las frecuencias de las variables cualitativas mediante la prueba de Chi-cuadrado y el test exacto de Fisher. Mediante el análisis multivariado de regresión logística se estimó los Odds Ratio (OR) ajustados. Se respetó el principio de confidencialidad.

\section{RESULTADOS}

Entre enero del 2009 y junio del 2014, nacieron un total de 745 prematuros de muy bajo peso en el Hospital Nacional Edgardo Rebagliati Martins; ingresaron al estudio, 190 recién nacidos que cumplieron los criterios de inclusión. Se excluyeron a 275 recién nacidos fallecidos, 50 referidos de otros hospitales, siete presentaron malformaciones congénitas mayores, 165 abandonaron el seguimiento antes de los dos años y 58 presentaron datos incompletos en sus respectivas historias clínicas.

El promedio del peso al nacimiento de los prematuros del estudio fue de $1.180,53 \pm 212,40$ gramos, con una edad gestacional en semanas de 29,86 $\pm 2,33$; el $51,58 \%$ fue género masculino, siendo la cesárea la vía de nacimiento en el $91,58 \%$ de los casos. El $44,21 \%$ de los recién nacidos prematuros de muy bajo peso presentó como antecedente materno a la preeclampsia; el 24,61\% fueron embarazos múltiples; mientras que la ruptura prematura de membranas y la corioamnionitis estuvieron presentes en el $27,37 \%$ y $8,95 \%$ de los casos, respectivamente. Los corticoides prenatales fueron aplicados al $83,68 \%$ de las gestantes con amenaza de parto prematuro.

Tabla 1. Características natales en los recién nacidos de muy bajo peso

\begin{tabular}{|c|c|c|c|c|c|}
\hline & Características & $\begin{array}{c}\text { Total } \\
(\mathrm{n}=190) \\
\mathrm{n}(\%)\end{array}$ & $\begin{array}{c}<1000 \mathrm{~g} \\
(\mathrm{n}=44) \\
\mathrm{n}(\%)\end{array}$ & $\begin{array}{c}>1000-1499 \mathrm{~g} \\
(\mathrm{n}=146) \\
\mathrm{n}(\%)\end{array}$ & $\mathrm{p}$ \\
\hline \multirow{2}{*}{ Género } & Masculino & $98(51,58)$ & $20(45,45)$ & $78(53,42)$ & 0,35 \\
\hline & Femenino & $92(48,42)$ & $24(54.55)$ & $68(46,58)$ & \\
\hline \multirow{2}{*}{ Edad gestacional } & $<28$ semanas & $28(14,74)$ & $22(50,00)$ & $6(4,11)$ & $<0,01$ \\
\hline & $>28$ semanas & $162(85,26)$ & $22(50,00)$ & $140(95,89)$ & \\
\hline \multirow{2}{*}{ Tipo de parto } & Vaginal & $16(8,42)$ & $8(18,18)$ & $8(5,48)$ & $<0,01$ \\
\hline & Cesárea & $174(91,58)$ & $36(81,82)$ & $138(94,52)$ & \\
\hline \multirow{2}{*}{$\begin{array}{c}\text { Apgar }<7 \text { a los } 5 \\
\text { minutos }\end{array}$} & Sí & $13(6,84)$ & $6(13,64)$ & $7(4,79)$ & 0,04 \\
\hline & No & $177(93,17)$ & $38(86,36)$ & $139(95,21)$ & \\
\hline \multirow{2}{*}{$\begin{array}{l}\text { Requirió } \\
\text { reanimación } \\
\text { neonatal }\end{array}$} & Sí & $69(36,32)$ & $23(52,27)$ & $46(31,51)$ & 0,01 \\
\hline & No & $121(63,68)$ & $21(47,73)$ & $100(68,49)$ & \\
\hline
\end{tabular}

Fuente: Datos tomados de las historias clínicas del Servicio de Neonatología del HNERM

Los recién nacidos con peso menor a 1.000 gramos fueron el $23,16 \%$, encontrándose una diferencia significativa frente a los que pesaron de 1.000 a 1.499 gramos según el tipo de parto $(p<0,01)$. En relación al puntaje de Apgar al minuto, el
$13,64 \%$ de los recién nacidos que tuvieron menos de 1.000 gramos presentaron un puntaje menor a 7 a los 5 minutos ( $p=0,04)$; el parto vaginal fue el $18,18 \%$ y el $52,27 \%$ necesitaron reanimación neonatal, como se describe en la Tabla 1. 
Tabla 2. Morbilidades post natales de los recién nacidos de muy bajo peso

\begin{tabular}{|c|c|c|c|c|c|}
\hline & Características & $\begin{array}{c}\text { Total } \\
(\mathrm{n}=190) \\
\mathrm{n}(\%)\end{array}$ & $\begin{array}{c}<1000 \mathrm{~g} \\
(\mathrm{n}=44) \\
\mathrm{n}(\%)\end{array}$ & $\begin{array}{c}>1000-1499 \mathrm{~g} \\
(\mathrm{n}=146) \\
\mathrm{n}(\%)\end{array}$ & $\mathrm{p}$ \\
\hline \multirow{2}{*}{ Sepsis precoz } & Sí & $139(73,16)$ & $37(84,09)$ & $102(69,86)$ & 0,06 \\
\hline & No & $51(26,84)$ & $7(15,91)$ & $44(30,14)$ & \\
\hline \multirow{2}{*}{$\begin{array}{l}\text { Persistencia de } \\
\text { conducto arterioso }\end{array}$} & Sí & $101(53,16)$ & $30(68,18)$ & $71(48,63)$ & 0,02 \\
\hline & No & $89(46,84)$ & $14(31,82)$ & $75(51,37)$ & \\
\hline \multirow{2}{*}{$\begin{array}{l}\text { Enfermedad } \\
\text { membrana hialina }\end{array}$} & Sí & $93(48,95)$ & $31(70,45)$ & $62(42,27)$ & $<0,01$ \\
\hline & No & $97(51,05)$ & $13(29,55)$ & $84(57,53)$ & \\
\hline \multirow{2}{*}{ Sepsis tardia } & Sí & $78(41,05)$ & $26(59,09)$ & $52(35,62)$ & $<0,01$ \\
\hline & No & $112(51,05)$ & $18(40,91)$ & $94(64,38)$ & \\
\hline \multirow{2}{*}{$\begin{array}{c}\text { Displasia } \\
\text { broncopulmonar }\end{array}$} & Sí & $50(26,32)$ & $30(68,18)$ & $20(13,70)$ & $<0,01$ \\
\hline & No & $140(73,68)$ & $14(31,82)$ & $126(86,30)$ & \\
\hline \multirow{2}{*}{$\begin{array}{l}\text { Hemorragia } \\
\text { intraventricular }\end{array}$} & Sí & $44(23,16)$ & $14(31,82)$ & $30(20,55)$ & 0,12 \\
\hline & No & $146(76,84)$ & $30(68,18)$ & $116(79,45)$ & \\
\hline \multirow{2}{*}{$\begin{array}{l}\text { Enterocolitis } \\
\text { necrotizante }\end{array}$} & Sí & $25(13,16)$ & $9(20,45)$ & $16(10,96)$ & 0,10 \\
\hline & No & $165(86,84)$ & $35(79,54)$ & $130(89,04)$ & \\
\hline
\end{tabular}

Fuente: Datos tomados de las historias clínicas del Servicio de Neonatología del HNERM

El 68,18\% de los recién nacidos con un peso menor de 1.000 gramos presentó persistencia del conducto arterioso frente a un $48,63 \%$ en los recién nacidos que pesaron de 1.000 a 1.499 $(p=0,02)$. El 70,45\% desarrolló enfermedad de la membrana hialina en el grupo menor a 1.000 gramos de peso, frente a un $42,27 \%$ en el grupo de 1.000 a 1.499 gramos $(p<0,01)$. Asimismo, el $59,09 \%$ presentó sepsis tardía y el $68,18 \%$ displasia broncopulmonar, como se describe en la Tabla 2.

Tabla 3. Relación entre los antecedentes maternos y neonatales con la presencia de alteraciones neurosensoriales y del desarrollo psicomotor de los recién nacidos de muy bajo peso

\begin{tabular}{|c|c|c|c|c|c|c|c|c|}
\hline & $\begin{array}{c}\text { Retinopatía } \\
\text { de la } \\
\text { prematuridad } \\
(n=48) \\
n(\%)\end{array}$ & $\mathrm{p}$ & $\begin{array}{c}\text { Hipoacusia } \\
\text { neurosensorial } \\
(n=26) \\
n(\%)\end{array}$ & $\mathrm{p}$ & $\begin{array}{c}\text { Alteración } \\
\text { del } \\
\text { desarrollo } \\
\text { psicomotor } \\
(\mathrm{n}=81) \\
\mathrm{n}(\%)\end{array}$ & $\mathrm{p}$ & $\begin{array}{c}\text { Parálisis } \\
\text { cerebral } \\
\\
\left(\begin{array}{c}n=7) \\
n(\%)\end{array}\right.\end{array}$ & $\mathrm{p}$ \\
\hline \multicolumn{9}{|l|}{ Género } \\
\hline Masculino & $29(60,42)$ & 0,16 & $12(46,15)$ & 0,55 & $41(50,60)$ & 0,82 & $1(1,02)$ & 0,04 \\
\hline Femenino & $19(39,58)$ & & $14(53,85)$ & & $40(49,40)$ & & $6(6,52)$ & \\
\hline \multicolumn{9}{|l|}{ Peso } \\
\hline$<1000 \mathrm{~g}$ & $22(45,83)$ & $<0,01$ & $7(15,91)$ & 0,62 & $23(28,40)$ & 0,14 & $0(0.00)$ & 0,14 \\
\hline$\geq 1000 \mathrm{~g}$ & $26(54,17)$ & & $19(13,10)$ & & $58(71,60)$ & & $7(4,79)$ & \\
\hline \multicolumn{9}{|l|}{ Edad gestacional } \\
\hline $\begin{array}{c}<28 \\
\text { semanas }\end{array}$ & $17(35,42)$ & $<0,01$ & $5(19,23)$ & 0,49 & $16(19,75)$ & 0,09 & $0(0,00)$ & 0,26 \\
\hline $\begin{array}{c}\geq 28 \\
\text { semanas }\end{array}$ & $31(64,58)$ & & $21(80,77)$ & & $65(80,25)$ & & $7(4,32)$ & \\
\hline \multicolumn{9}{|c|}{ Pequeño para edad gestacional } \\
\hline Sí & $7(14,58)$ & $<0,01$ & $8(30,77)$ & 0,92 & $23(38,33)$ & 0,42 & $1(1,67)$ & 0,32 \\
\hline No & $41(85,42)$ & & $18(69,23)$ & & $58(44,62)$ & & $6(4,62)$ & \\
\hline \multicolumn{9}{|l|}{ Parto vaginal } \\
\hline Sí & $4(8,33)$ & 0,98 & $4(15,38)$ & 0,17 & $10(62,50)$ & 0,09 & $1(6,25)$ & 0,57 \\
\hline No & $44(91,67)$ & & $22(84,62)$ & & $71(40,80)$ & & $6(3,45)$ & \\
\hline
\end{tabular}


Secuelas del neurodesarrollo de recién nacidos prematuros de extremadamente bajo peso y de muy bajo peso a los dos años de edad, egresados de la Unidad de Cuidados Intensivos Neonatales del Hospital Nacional Edgardo Rebagliati Martins 2009-2014

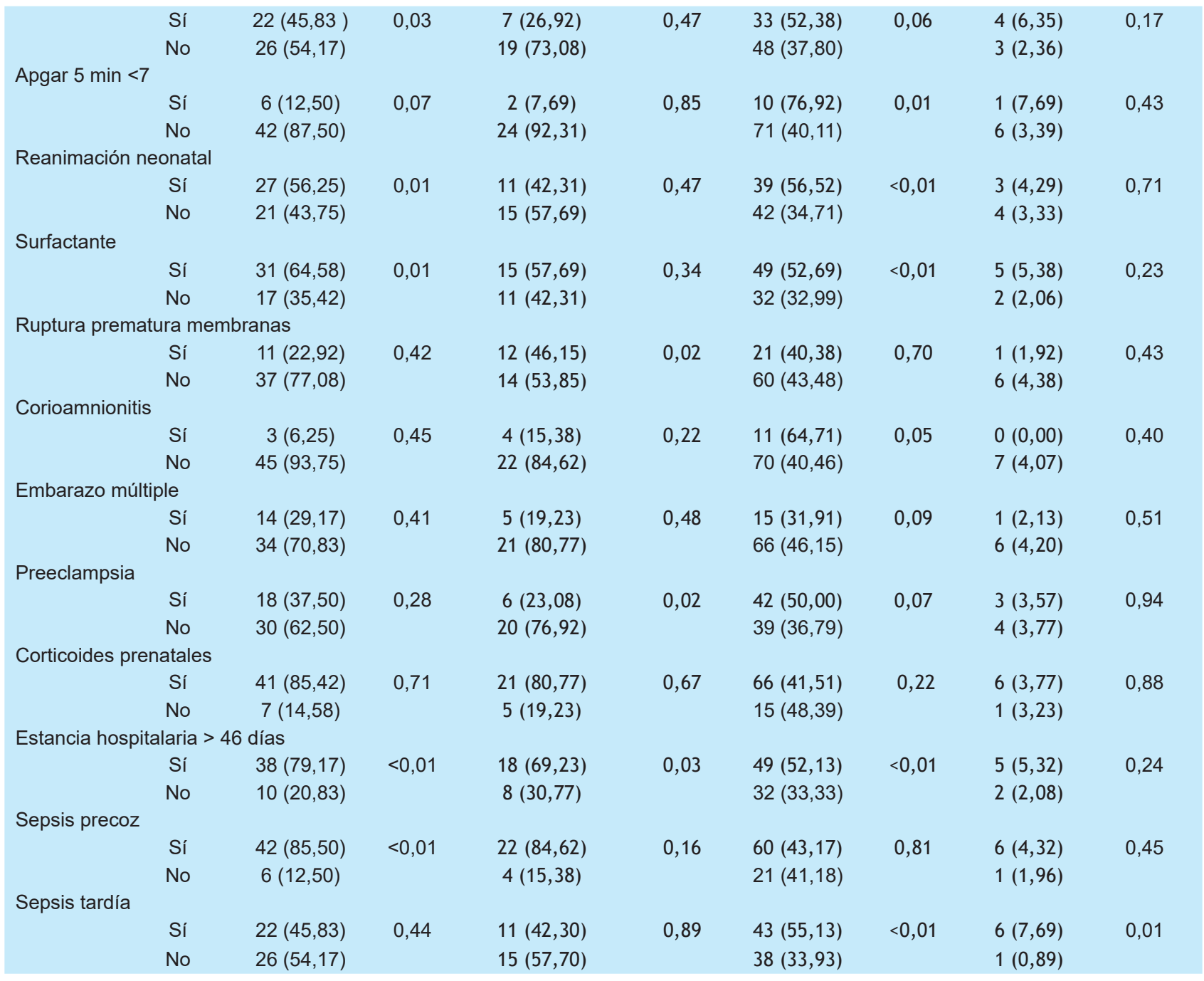

Fuente: Datos tomados de las historias clínicas del Servicio de Neonatología HNERM

La alteración del desarrollo psicomotor se presentó en el $42,63 \%$ de los prematuros de muy bajo peso al nacer. Siendo la necesidad de reanimación neonatal, el requerimiento de aplicación de surfactante, el diagnóstico de sepsis tardía y la estancia hospitalaria mayor a 46 días, fueron las características asociadas a la presencia de alteración del desarrollo psicomotor $(\mathrm{p}<0,01)$.
La retinopatía de la prematuridad se halló en $25,26 \%$ y la hipoacusia neurosensorial en $13,68 \%$ en la población de estudio. En el análisis de las características perinatales como el peso al nacer menor a 1.000 gramos, edad gestacional menor a 28 semanas, pequeño para la edad gestacional, estancia hospitalaria mayor a 46 días, sepsis precoz, Apgar menor de 7 al minuto, se logró observar una asociación con la presencia de retinopatía de la prematuridad $(p<0,01)$. 
Tabla 4. Presencia de alteraciones del neurodesarrollo a los dos años de seguimiento de los prematuros de muy bajo peso al nacer

\begin{tabular}{|c|c|c|c|c|}
\hline Características & $\begin{array}{c}\text { Total } \\
(\mathrm{n}=190) \\
\mathrm{n}(\%)\end{array}$ & $\begin{array}{c}<1000 \mathrm{~g} \\
(\mathrm{n}=44) \\
\mathrm{n}(\%)\end{array}$ & $\begin{array}{c}>1000-1499 \mathrm{~g} \\
(\mathrm{n}=146) \\
\mathrm{n}(\%)\end{array}$ & $\mathrm{p}$ \\
\hline \multicolumn{5}{|l|}{ Retraso del desarrollo psicomotor } \\
\hline Sí & $81(42,63)$ & $23(52,27)$ & $58(39,73)$ & 0,14 \\
\hline No & $109(57,37)$ & $21(47,73)$ & $88(60,27)$ & \\
\hline \multicolumn{5}{|l|}{ Retinopatía de la prematuridad } \\
\hline Sí & $48(25,26)$ & $22(50,00)$ & $26(17,81)$ & $<0,01$ \\
\hline No & $142(74,74)$ & $22(50,00)$ & $120(82,19)$ & \\
\hline \multicolumn{5}{|l|}{ Hipoacusia neurosensorial } \\
\hline Sí & $26(13,68)$ & $7(15,91)$ & $19(13,01)$ & 0,62 \\
\hline No & $164(86,32)$ & $37(84,09)$ & $127(86,99)$ & \\
\hline \multicolumn{5}{|l|}{ Parálisis cerebral } \\
\hline Sí & $7(3,68)$ & $0(0,00)$ & $7(4,79)$ & 0,14 \\
\hline No & $183(96,32)$ & $0(0,00)$ & $139(95,21)$ & \\
\hline \multicolumn{5}{|l|}{ Síndrome convulsivo } \\
\hline Sí & $7(3,68)$ & $1(2,27)$ & $6(4,11)$ & 0,57 \\
\hline No & $183(96,32)$ & $43(97,73)$ & $140(95,89)$ & \\
\hline
\end{tabular}

Fuente: Datos tomados de las historias clínicas del Servicio de Neonatología del HNERM

En relación a las alteraciones del neurodesarrollo a los dos años de edad, se confirmó la presencia de retinopatía de la prematuridad en el $50 \%$ de los recién nacidos con peso menor a 1.000 gramos $(p<0,01)$. Así como el $52,27 \%$ de este grupo de recién nacidos presentó alteración del desarrollo psicomotor, pero sin significación estadística, como se describe en la Tabla 4.

\section{DISCUSIÓN}

En la población de estudio de 190 recién nacidos prematuros de muy bajo peso, se encontró como antecedentes maternos a la preeclampsia en 84 de ellos (44,21\%), a la ruptura prematura de membranas 52 (27,37\%), al embarazo múltiple $47(24,61 \%)$ y a la corioamnionitis $17(8,95 \%)$, sin ninguna diferencia significativa. El estudio realizado por Wang reportó los mismos antecedentes a pesar de que el estudio se realizó con una población de 109 recién nacidos extremadamente prematuros ${ }^{(16)}$.

Las morbilidades que se encontraron en los recién nacidos de muy bajo peso fueron la enfermedad de membrana hialina, sepsis tardía, displasia broncopulmonar en los recién nacidos con peso menor a 1.000 gramos, cuando se compararon con los de 1.000 a 1.499 gramos $(p<0,01)$. García-Muñoz y colaboradores reportaron una incidencia de sepsis tardía de $50,5 \%$ en los menores de 26 semanas y estuvo asociada con la prolongada estancia hospitalaria, similar a nuestros resultados $(41,05 \%)$ para la sepsis tardía ${ }^{(17)}$.
Los recién nacidos que presentaron retinopatía de la prematuridad fueron 48 (25,26\%), siendo la edad gestacional menor de 28 semanas al nacer y el peso menor a 1.000 gramos; antecedentes similares reportados por Port y Yau ${ }^{(18,19)}$. En el estudio realizado por Fernández R, se reportó 30,8\% para los menores de 1.500 gramos al nacer ${ }^{(20)}$. Los resultados evidencian una asociación entre la retinopatía con aquellos recién nacidos que fueron sometidos a reanimación neonatal, ser pequeño para la edad gestacional, estancia hospitalaria mayor de 46 días y el uso de surfactante $(p=<0,01)$; el estudio realizado por Port reportó los mismos factores y otros, como la displasia broncopulmonar y enterocolitis necrotizante, no mostrando diferencias significativas con el peso de los recién nacidos estudiados ${ }^{(18)}$. Asimismo, se asociaría a mayor morbilidad y alteración en el neurodesarrollo de este grupo de recién nacidos con diagnóstico de retinopatía, según lo reportado por Beligere y Medina ${ }^{(21,22)}$.

La detección de hipoacusia neurosensorial en el grupo de estudio fue de $13,68 \%$, este valor es hasta 40 veces más alto que en la población general sin factores de riesgo, donde se describe una incidencia de 3 a 5 casos por cada mil neonatos. La frecuencia de hipoacusia neurosensorial se encuentra en valores superiores comparado con los estudios realizados por García y Moore que reportaron una frecuencia de $0,4 \%{ }^{(23,24)}$, la incidencia en el estudio EPICURE fue del 0,2\% para hipoacusia grave o severa con una población de neonatos menores de 23 semanas. Actualmente no se cuenta con investigaciones nacionales en los últimos cinco años. 
Secuelas del neurodesarrollo de recién nacidos prematuros de extremadamente bajo peso y de muy bajo peso a los dos años de edad, egresados de la Unidad de Cuidados Intensivos Neonatales del Hospital Nacional Edgardo Rebagliati Martins 2009-2014

Son propuestos múltiples factores implicados en la patogenia de la hipoacusia en la población de estudio, la ruptura prematura de membranas obtuvo una asociación significativa $(p=0,02)$. El estudio realizado por PeñalozaLópez describió a la infección materna como un factor de riesgo importante, resultados que coinciden con el presente estudio ${ }^{(25)}$. Asimismo, otras morbilidades neonatales, como la sepsis neonatal y la presencia de hemorragia intracraneana, están relacionados con la presencia de hipoacusia, como los reportados por Borkoski, Bielecki y Nuñez-Batalla ${ }^{(26-28)}$. Los resultados del presente estudio evidenciaron que, mientras más días de permanencia del recién nacido en la Unidad de Cuidados intensivos, mayor fue la frecuencia de la presencia de hipoacusia neurosensorial, tendencia que coincide con el estudio presentado por Peñaloza- López ${ }^{(25)}$.

Con respecto al retraso del desarrollo psicomotor, este se representó en 81 casos $(42,43 \%$ de la población de estudio); asimismo, se encontró el Apgar menor a 7 a los cinco minutos en $76,92 \%$ de la población, resultado que tiene similitud con el estudio de Wang ${ }^{(16)}$ donde reporta que el Apgar menor o igual a 3 a los cinco minutos fue un predictor para la sobrevida y morbilidad en los prematuros menores de 26 semanas al nacer. Asimismo, los recién nacidos que recibieron reanimación durante su atención inmediata y presentaron sepsis tardía tuvieron mayor riesgo de presentar algún tipo de alteración del desarrollo psicomotor $(p<0,01)$, similar a lo reportado por Rand, Inder $\mathrm{y} \operatorname{otros}^{(9)}$.

A pesar del impacto de los avances en el área de la neonatología, en referencia a la disminución de la mortalidad infantil y neonatal, no se constata una disminución significativa en la incidencia de parálisis cerebral infantil $(\mathrm{PCl})$, esto debido a que no existe una causa específica de la $\mathrm{PCl}$, sino que puede sobrevenir por distintos factores que tienen lugar antes, durante o después del nacimiento.

El estudio realizado por Hirvonen reportó una menor tasa de incidencia de $\mathrm{PCl}$ en neonatos prematuros menores de 26 semanas de gestación ${ }^{(29)}$. Sin embargo, en el presente estudio no se encontraron casos de $\mathrm{PCl}$ en prematuros extremos, resultado que concuerda con el estudio de Himmelmann $\mathrm{K}^{(30)}$. El ser pretérmino sería un factor de riesgo para presentar alteraciones neurológicas como los que describen los estudios realizados por Silva y Suppiej ${ }^{(31,32)}$. No obstante, las complicaciones perinatales son factores determinantes para la mortalidad de estos extremos prematuros según Taboada-Lugo ${ }^{(33)}$.

En el presente estudio, se evidencia una mayor incidencia de $\mathrm{PCl}$ en el sexo femenino, resultado que se contrapone por lo descrito en el estudio de Schlapbach $\mathrm{LJ}^{(34)}$, en donde se demuestra un discreto predominio del género masculino, que se asocia significativamente con el resultado adverso y desfavorable. Por otra parte, la hemorragia intraventricular se ha visto asociada con mayor riesgo de presentar $\mathrm{PCl}$, resultado que coincide con lo descrito por Schlapbach.
En conclusión, el retraso del desarrollo psicomotor y la retinopatía fueron las complicaciones más importantes que presentaron los recién nacidos prematuros de extremadamente bajo peso y los recién nacidos prematuros de muy bajo a los dos años de edad.

\section{REFERENCIAS BIBLIOGRÁFICAS}

1. March of Dimes, PMNCH, Save the Children, WHO. Born too soon: The Global Action Report on Preterm Birth. Howson CP, Kinney MV, Lawn JE, eds. Ginebra: World Health Organization, 2012.

2. Blencowe $\mathrm{H}$, Cousens S, Oestergaard MZ, Chou D, Moller AB, Narwal R, et al. National, regional, and worldwide estimates of preterm birth rates in the year 2010 with time trends since 1990 for selected countries: a systematic analysis and implications. Lancet. 2012; 379 (9832):2162-72.

3. Liu L, Oza S, Hogan D, Perin J, Rudan I, Lawn J, et al. Global, regional, and national causes of child mortality in 2000-13, with projections to inform post-2015 priorities: an updated systematic analysis. Lancet. 2015; 385 (9966):430-40.

4. Lawn J, Davidge R, Paul V, von Xylander S, de Graft Johnson J, Costello A, et al. Born too soon: care for the preterm baby. Reprod Health. 2013; 10(1):S5.

5. Mathews T, MacDorman M. Infant mortality statistics from the 2010 period linked birth/infant death data set. Natl Vital Stat Rep. 2013; 62(8):1-26.

6. Barros F, Barros A, Villar J, Matijasevich A, Domingues M, Victora C. How many low birthweight babies in low- and middle-income countries are preterm?. Rev Saude Pública. 2011; 45 (3):607-16

7. Vohr B. Neurodevelopmental outcomes of extremely preterm infants. Clin Perinatol.2014; 41 (1):241-55.

8. Volpe J, Kinney H, Jensen F. The developing oligodendrocyte: key cellular target in brain injury in the premature infant. Int J Dev Neurosci. 2011; 29 (4):423-40.

9. Rand K, Austin N, Inder T, Bora S, Woodward L. Neonatal infection and later neurodevelopmental risk in the very preterm infant. J Pediatr.2016; 170:97-104.

10. O’Shea T, Joseph R, Kuban K, Allred E, Ware J, Coster T, et al. Elevated blood levels of inflammation-related proteins are associated with an attention problem at age 24 mo in extremely preterm infants. Pediatr Res. 2014; 75 (6):781-7.

11. Brunkhorst J, Weiner J, Lantos J. Infants of borderline viability: the ethics of delivery room care. Semin Fetal Neonatal Med. 2014; 19 (5):290-5.

12. Schmidt B, Anderson PJ, Doyle LW, Dewey D, Grunau RE, Asztalos EV, et al. Survival without disability to age 5 years after neonatal caffeine therapy for apnea of prematurity. JAMA. 2012; 307 (3):275-82.

13. Kawaza K, Machen H, Brown J, Mwanza Z, Iniguez S, Gest A, et al. Efficacy of a low-cost bubble CPAP system in treatment of respiratory distress in a neonatal ward in Malawi. PLoS One. 2014; 9 (1):1-8.

14. Arreola-Ramírez G, Fernández-Carrocera LA, Ramírez-López $\mathrm{CZ}$, Barrera-Reyes RH, Regil-Vélez LM, Ríos-Flores B, et al. Desarrollo neurológico en el primer año de vida de infantes prematuros con peso menor a 1,500 g en una institución de tercer nivel. Perinatol Reprod Hum. 2011; 25 (3):146-54.

15. Barra L, Alvarado R. Frecuencia de riesgo neurobiológico en recién nacidos. Rev Chil Pediatr. 2012; 83(6):552-62.

16. Wang Y, Tanbo T, Ellingsen L, Abyholm T, Henriksen T. Effect of pregestational maternal, obstetric and perinatal factors 
on neonatal outcome in extreme prematurity. Arch Gynecol Obstet. 2011; 284 (6):1381-7.

17. García-Muñoz F, García-Alix A, García J, Figueras J. Morbidity and mortality in newborns at the limit of viability in Spain: a population-based study. An Pediatr (Barc). 2014; 80 (6):34856.

18. Port A, Chan R, Ostmo S, Choi D, Chiang M. Risk factors for retinopathy of prematurity: insights from outlier infants. Graefes Arch Clin Exp Ophthalmol. 2014; 252 (10): 1669-77.

19. Yau G, Lee J, Tam V, Yip S, Cheng E, Liu C, et al. Incidence and risk factors for retinopathy of prematurity in multiple gestations: a chinese population study. Medicine (Baltimore). 2015; 94 (18):e867.

20. Fernández R, D’Apremont I, Domínguez A., Tapia J, Red Neonatal Neocosur. Supervivencia y morbilidad en recién nacidos de muy bajo peso al nacer en una Red Neonatal sudamericana. Arch Argent Pedriatr. 2014; 112 (5):405-12.

21. Beligere N, Perumalswamy V, Tandon M, Mittal A, Floora J, Vijayakumar B, et al. Retinopathy of prematurity and neurodevelopmental disabilities in premature infants. Semin Fetal Neonatal Med. 2015; 20 (5):346-53.

22. Medina M, Kahn I, Muñoz P, Leyva J, Moreno J, Vega S. Neurodesarrollo infantil: características normales y signos de alarma en el niño menor de cinco años. Rev Peru Med Exp Salud Pública. 2015; 32 (3):565-73.

23. García P, San Feliciano L, Benito F, García R, Guzmán J, Salas $S$, et al. Evolución a los 2 años de edad corregida de una cohorte de recién nacidos con peso inferior o igual a $1.500 \mathrm{~g}$ de los hospitales pertenecientes a la red neonatal SEN1500. An Pediatr. 2013; 79 (5):279-87.

24. Moore T, Hennessy E, Myles J, Johnson S, Draper E, Costeloe K, et al. Neurological and developmental outcome in extremely preterm children born in England in 1995 and 2006: the EPICure studies. BMJ. 2012; 345:e7961.

25. Peñaloza-López Y, García-Pedroza F, Castillo-Maya G, JiménezPérez J. Hipoacusia-sordera congénita y su relación con el peso bajo al nacimiento en México y en algunos otros países. Rev Mex AMCAOF. 2012; 1 (2):82-9.

26. Borkoski S, Falcón J, Bueno J, Pérez J, López Z, Ramos Á. Resultados de un programa de detección precoz de la hipoacusia neonatal. Acta Otorrinolaringológica Española. 2013; 64(2):92-6.

27. Bielecki I, Horbulewicz A, Wolan T. Risk factor associated with hearing loss in infants: an analysis of 5282 referred neonates. Int J Pediatr Otorhinolaryngol. 2011;75(7):925-30.

28. Nuñez-Batalla F, Trinidad-Ramos G, Sequí-Canet J, Alzina de
Aguilar V, Jáudenes-Casaubón C. Indicadores de riesgo de hipoacusia neurosensorial infantil. Acta Otorrinolaringologica Española. 2012;63(5): 382-90.

29. Hirvonen M, Ojala R, Korhonen P, Haataja P, Eriksson K, Gissler $M$, et al. Cerebral palsy among children born moderately and late preterm. Pediatrics. 2014;134(6):1584-93.

30. Himmelmann K, Uvebrant P. The panorama of cerebral palsy in Sweden. XI. Changing patterns in the birth-year period 2003-2006. Acta Paediatr. 2014; 103(6):618-24.

31. da Silva A, Tavares L, da Silva J, Ribeiro L. Factors related to motor developmental delay of newborns. Journal of Human Growthand Development. 2014; 24 (3):320-7.

32. Suppiej A, Franzoi M, Vedovato S, Marucco A, Chiarelli S, Zanardo V. Neurodevelopmental outcome in preterm histological chorioamnionitis. Early Hum Dev. 2009; 85 (3):187-9.

33. Taboada-Lugo N, Quintero-Escobar K, Casamajor-Castillo M,González-Torres K, Marrero-Infante J, Cruz-Ubeda S, et al. Epidemiología de la parálisis cerebral en el Estado Plurinacional de Bolivia, 2009-2012. Rev Peru de Epidemiol. 2013; 17 (2):1-7.

34. Schlapbach L, Adams M, Proietti E, Aebischer M, Grunt S, Borradori-Tolsa $C$, et al. Outcome at two years of age in a Swiss national cohort of extremely preterm infants born between 2000 and 2008. BMC Pediatr. 2012; 12: 198.

Fuentes de financiamiento:

Este artículo ha sido financiado por los autores.

\section{Conflictos de interés:}

Los autores declaran no tener ningún conflicto de interés.

\section{Correspondencia:}

Carmen Luisa Fernández Sierra

Dirección: Avenida Horacio Urteaga 752 Dpto. 206 Jesús María

Lima-11 Perú

Teléfono: 511-985 494858

Correo electrónico: fernandezsierracarmen@yahoo.es

Recibido: 20 de setiembre de 2016 Aprobado: 15 de enero de 2017 